\title{
Numerical modelling of error field penetration
}

\author{
Q. Yu ${ }^{1}$, S. Günter ${ }^{1}$, Y. Kikuchi ${ }^{2,3}$ and K.H. Finken ${ }^{2}$ \\ ${ }^{1}$ Max-Planck-Institut für Plasmaphysik, EURATOM Association, 85748 Garching, Germany \\ ${ }^{2}$ Institut für Plasmaphysik, Forschungszentrum Jülich, 52425 Jülich, Germany
}

\begin{abstract}
Error field (or externally applied helical field) penetration is studied numerically based on the two fluids equations. It is shown that there is a minimum in the required field amplitude when the applied helical field frequency is the same as the mode frequency being determined by both the background equilibrium plasma rotation and the diamagnetic drift. The mode penetration threshold significantly increases as the field frequency deviates from the mode frequency and can become asymmetric on the two sides of the minimum due to parallel heat transport. After mode penetration the nonlinear saturated island width is found to be smaller for a larger electron diamagnetic drift frequency.
\end{abstract}

PACS numbers: 52.35.Py, 52.35.Vd, 52.55.Tn, 52.35.-y

\section{Introduction}

The effect of an externally applied resonant helical field or an error field on the magnetic islands is of great interest for magnetic fusion research. When the island is large enough, it will be locked to the externally applied helical field [1,2]. Even if without an external helical field, the helical current induced in the vacuum vessel by an unstable mode will produce a helical field to act back on the island and to lead to the mode locking of a large island [1]. For the plasma being originally stable to the tearing mode, an externally applied resonant field can penetrate through the resonant surface, generating a magnetic island there (mode penetration) [2-7]. Both experimental and theoretical studies have been carried out for understanding the mode penetration physics. The experimental results show that the penetration threshold of an error field is very small, typically $b_{r}(r=a) / B_{0 \mathrm{t}} \sim 10^{-4}-10^{-3}$ on existing tokamaks, where $b_{r}(r=a)$ is the radial magnetic field perturbation at the plasma edge $r=a$ and $B_{0 \mathrm{t}}$ is the equilibrium toroidal magnetic field [2-7]. The mode penetration threshold is expected to be significantly lower for a fusion reactor, especially during the plasma current rise phase with a low plasma density and rotation velocity [2-6]. For a higher $\beta$ plasma, the mode penetration threshold is found to be lower on DIII-D possibly due to the destabilizing role of the perturbed bootstrap current $[5,6]$. Recently it was shown on TEXTOR that the relative frequency between the mode and the applied helical field is important in determining the threshold for the mode onset $[8,9]$. When these two frequencies are the same, the mode

3 Present address: Graduate School of Engineering, University of Hyogo, 2167 Syosha, Himeji, 671-2201 Hyogo, Japan. penetration threshold reaches a minimum value. The required helical field amplitude for mode penetration significantly increases if these two frequencies are different $[8,9]$.

Most theoretical results on mode penetration are based on reduced MHD equations. In this limit it has been shown that the penetration threshold of a static helical field is higher for a larger equilibrium plasma rotation frequency $\omega$ due to the shielding current at the mode rational surface. In addition, the penetration threshold also depends on the plasma resistive and viscose diffusion time $\tau_{\mathrm{R}}$ and $\tau_{\mu}$ and the Alfven time $\tau_{\mathrm{A}}$ [10-13]. Based on the nonlinear modification of the equilibrium plasma rotation velocity and the nonlinear island growth equation (Rutherford equation), the required $b_{r}$ for mode penetration is found to be proportinoal to $\omega^{4 / 3}$ when the plasma inertia effect is more important than the viscous one and to $\omega^{2 / 5}$ in the opposite limit $[2,10]$. If the linear response in the inner tearing region around the rational surface instead of the Rutherford equation is utilized, various different regimes are found $[11,12]$. Among them the so-called viscoresistive regimes are more relevant to tokamak plasmas. In this regime the penetration threshold $b_{r} / B_{0 \mathrm{t}}$ is proportional to $\omega \tau_{\mathrm{R}}\left(\tau_{\mathrm{R}} / \tau_{\mu}\right)^{7 / 12}\left(\tau_{\mathrm{A}} / \tau_{\mathrm{R}}\right)^{7 / 6}[11,12]$. For a fusion reactor the mode penetration is predicted to occur when $b_{r} / B_{0 \mathrm{t}}=2 \times 10^{-5}$ at the rational surface [12], being much lower than that of the existing tokamaks. This implies that mode penetration is a very important issue, and improved understanding of the relevant physics is necessary. Recently, results from the four-field model including the diamagnetic drift were obtained [14,15]. In [14] an analytic theory is constructed in a similar way to MHD theory $[11,12]$, and the diamagnetic drift is taken into account in the inner tearing region. The mode penetration 
threshold obtained from the two fluids equations is found to be similar to that from the reduced MHD equations, if the mode frequency due to the background plasma rotation is replaced by that due to the diamagnetic drift [14]. This is as expected since the nonlinear change in the diamagnetic drift frequency due to the parallel heat conduction is neglected. In [15] linear numerical results from the four-field model show that a sharp resonance between the error field and the mode occurs when the field frequency is the same as the mode frequency. Both the background plasma rotation and the diamagnetic drift are important in determining the mode penetration, since they could either increase or decrease the total mode frequency, depending on the plasma rotation direction [15]. Such a numerical result agrees with analytical theory [10].

Motivated by recent TEXTOR experimental results [8,9], in this paper the mode penetration is studied numerically using the (reduced) nonlinear two fluids equations in the periodical cylinder geometry. Both parallel and perpendicular transports are self-consistently included, being necessary for modelling the change in the diamagnetic drift frequency and the associated ion polarization current during the island growth driven by the error field. The nonlinear numerical results provide a better understanding of the mode penetration physics, showing the asymmetry of the penetration threshold on the two sides of the minimum value as observed in TEXTOR experiments $[8,9]$. In addition, the penetrated island width is found to decrease for a larger electron diamagnetic drift frequency, indicating a stabilizing role of the ion polarization current.

\section{Computational model}

A large aspect-ratio tokamak approximation is utilized in our model. The magnetic field is defined as $\boldsymbol{B}=B_{0 \mathrm{t}} \boldsymbol{e}_{\mathrm{t}}-$ $(k r / m) B_{0 \mathrm{t}} \boldsymbol{e}_{\theta}+\nabla \psi \times \boldsymbol{e}_{\mathrm{t}}$, where $\psi$ is the helical flux function, $m / r$ and $k=n / R$ are the wave vectors in $\boldsymbol{e}_{\theta}$ (poloidal) and $\boldsymbol{e}_{\mathrm{t}}$ (toroidal) directions, respectively, $R$ is the major radius, $m$ and $n$ are the poloidal and toroidal mode numbers of the mode and the subscript 0 denotes an equilibrium quantity. The plasma velocity is defined as $\boldsymbol{v}=v_{\|} \boldsymbol{e}_{\|}+\nabla \phi \times \boldsymbol{e}_{\mathrm{t}}$, where $\phi$ is the stream function.

The two fluids equations utilized here include the generalized Ohm's law, the equation of motion in the perpendicular (after taking $\nabla \times$ ) and parallel (to magnetic field) directions and the energy and mass conservation equations. Normalizing the length to the minor radius $a$, the time $t$ to the resistive time $\tau_{\mathrm{R}}=a^{2} \mu_{0} / \eta$, the helical flux $\psi$ to $a B_{0 \mathrm{t}}, \boldsymbol{v}$ to $a / \tau_{\mathrm{R}}$ and the electron temperature $T_{\mathrm{e}}$ and density $n_{\mathrm{e}}$ to their values at the magnetic axis, these equations become

$$
\begin{aligned}
& \frac{\mathrm{d} \psi}{\mathrm{d} t}=E-\eta j+\Omega\left(\nabla_{\|} n_{\mathrm{e}}+\nabla_{\|} T_{\mathrm{e}}\right), \\
& \frac{\mathrm{d} U}{\mathrm{~d} t}=-S^{2} \nabla_{\|} j+\mu \nabla_{\perp}^{2} U+S_{\mathrm{m}}, \\
& \frac{\mathrm{d} v_{\|}}{\mathrm{d} t}=-c_{\mathrm{s}}^{2} \nabla_{\|} P / n_{\mathrm{e}}+\mu \nabla_{\perp}^{2} v_{\|}, \\
& \frac{3}{2} n_{\mathrm{e}} \frac{\mathrm{d} T_{\mathrm{e}}}{\mathrm{d} t}=0.78(\Omega / \beta) \nabla_{\|} j+T_{\mathrm{e}} n_{\mathrm{e}} \nabla_{\|} v_{\|}+n_{\mathrm{e}} \nabla_{\|}\left(\chi_{\|} \nabla_{\|} T_{\mathrm{e}}\right) \\
& \quad+n_{\mathrm{e}} \nabla_{\perp}\left(\chi_{\perp} \nabla_{\perp} T_{\mathrm{e}}\right)+S_{\mathrm{p}}, \\
& \frac{\mathrm{d} n_{\mathrm{e}}}{\mathrm{d} t}=(\Omega / \beta) \nabla_{\|} j-\nabla_{\|}\left(n_{\mathrm{e}} v_{\|}\right)+\nabla_{\perp}\left(D_{\perp} \nabla_{\perp} n_{\mathrm{e}}\right)+S_{\mathrm{n}},
\end{aligned}
$$

where $\mathrm{d} / \mathrm{d} t=\partial / \partial t+\boldsymbol{v}_{\perp} \cdot \nabla_{\perp}, \nabla_{\|} f=(\boldsymbol{B} / B) \cdot \nabla f \approx$ $\left(\boldsymbol{B} / B_{0 \mathrm{t}}\right) \cdot \nabla f, \nabla_{\perp} f=f^{\prime}+(\partial f / \partial \theta) / r, \nabla_{\perp}^{2} f=\left[r\left(f^{\prime}\right)\right]^{\prime} / r-$ $\left(\partial^{2} f / \partial \theta^{2}\right) / r^{2}$ and the prime is for $\partial / \partial r . \quad j=\nabla_{\perp}^{2} \psi-$ $2 n B_{0 t} /(m R)$ being the toroidal plasma current density, $\eta$ is the normalized plasma resistivity, $E$ is the equilibrium electric field for maintaining the equilibrium plasma current density, $\Omega=\beta d_{1}$ determining the diamagnetic drift frequency, $\beta=4 n_{\mathrm{e}} T_{\mathrm{e}} / B^{2}, d_{1}=\omega_{\mathrm{ce}} / \nu_{\mathrm{e}}$ and $\omega_{\mathrm{ce}}$ and $\nu_{\mathrm{e}}$ are the electron cyclotron and collisional frequency, respectively. The magnetic Reynolds number $S=\tau_{\mathrm{R}} / \tau_{\mathrm{A}}$, where $\tau_{\mathrm{A}}=a / V_{\mathrm{A}}$ is the Alfven time. $U=-\nabla_{\perp}^{2} \phi$ being the plasma vorticity, $\mu$ is the plasma viscosity, $c_{\mathrm{s}}=\left(T_{\mathrm{e}} / m_{\mathrm{i}}\right)^{1 / 2}$ being the ion sound velocity and $p$ the plasma pressure. $\chi_{\|}$and $\chi_{\perp}$ are the parallel and perpendicular heat transport coefficients, $D_{\perp}$ is the perpendicular particle transport coefficient, $S_{\mathrm{p}}$ is the heating power, $S_{\mathrm{n}}$ is the particle source and $S_{\mathrm{m}}$ in equation (2) is the poloidal momentum source given by

$$
S_{\mathrm{m}}=\Omega_{\mathrm{E}}\left[1-(r / a)^{2}\right]^{3},
$$

which leads to an equilibrium poloidal plasma rotation.

Equations (1)-(5) are the coupled two fluids equations for modelling the nonlinear evolution of the drifting tearing mode [16], which reduce to the MHD equations if $\Omega=0$ is taken in Ohm's law to neglect the parallel electron temperature and the density gradient. The difference between the particle and the heat transport is taken into account, which is necessary for a small island for which the diamagnetic drift is important. Compared with previous theories, in equations (1)-(5) the two-dimensional electron heat transport is included selfconsistently. Cold ion assumption is made here, since the ion temperature is significantly lower than that of the electron's in these relevant experiments on TEXTOR $[8,9]$.

\section{Numerical results}

Equations (1)-(5) are solved simultaneously using the initial value code TM1, which has been used for modelling the linear stability of the drifting tearing mode earlier [16]. The modified version of this code has been used to study the nonlinear growth and saturation of the neoclassical tearing mode (NTM) and their stabilization by RF current $[17,18]$. Improved numerical methods are utilized in the code to reduce the numerical error for high values of $S$ and $\chi_{\|} / \chi_{\perp}[17,19,20]$.

The calculations are for a single helicity perturbation. In addition to the fundamental harmonic, higher harmonic perturbations as well as the change in the equilibrium quantities (the $m / n=0 / 0$ component) are self-consistently calculated. The toroidal magnetic field is taken to be a constant, so that the toroidal mode coupling is neglected. Fourier decomposition in the poloidal and toroidal directions and finite differences along the radial direction are utilized in the code. The calculation region is from the magnetic axis at $r=0$ to the plasma edge at $r=a$. The boundary conditions are as the following.

(a) The radial gradients of all quantities are zero at $r=0$.

(b) All the perturbations $(m / n \neq 0 / 0)$ are zero at $r=a$ except for the $m / n=2 / 1$ magnetic perturbation given by the following equation (7) to take into account the error field. 
(c) All the equilibrium $(m / m=0 / 0)$ quantities take the same value as the original equilibrium ones at $r=a$.

The effect of the error field is taken into account by the boundary condition

$$
\psi_{m / n}(r=a)=\psi_{\mathrm{a}} \cos \left(\omega_{\mathrm{f}} t+m \theta+n \phi\right),
$$

where $\psi_{\mathrm{a}}$ describes the applied helical field amplitude at $r=a$ of the $m / n$ component and $\omega_{\mathrm{f}}$ is the field frequency. The radial magnetic field is given by $b_{r, m / n}=m \psi_{m / n} / r$. Here the mode penetration of the $m / n=2 / 1$ mode is studied.

The input parameters are based on TEXTOR experimental parameters. The toroidal magnetic field is $B_{0 \mathrm{t}}=2.5 \mathrm{~T}$ and the plasma minor and major radii are $a=0.47 \mathrm{~m}$ and $R=1.75 \mathrm{~m}$, respectively. The equilibrium electron temperature and density profiles are modelled by

$$
\begin{aligned}
& T_{\mathrm{e}}=T_{\mathrm{e} 0}\left[1-(r / a)^{2}\right]+T_{\mathrm{ea}}, \\
& n_{\mathrm{e}}=n_{\mathrm{e} 0}\left[1-(r / a)^{2}\right]+n_{\mathrm{ea}},
\end{aligned}
$$

where $T_{\mathrm{e} 0}=1800 \mathrm{eV}, T_{\mathrm{ea}}=300 \mathrm{eV}, n_{\mathrm{e} 0}=3.2 \times 10^{19} \mathrm{~m}^{-3}$ and $n_{\text {ea }}=3.0 \times 10^{18} \mathrm{~m}^{-3}[8,13,15]$. These lead to the normalized parameters $S=1.97 \times 10^{8}, \Omega=6.3 \times 10^{4}, c_{\mathrm{s}}=1.2 \times 10^{7}$, $d_{1}=2.5 \times 10^{7}$ and $\chi_{\|}=1.1 \times 10^{9}\left(a^{2} / \tau_{\mathrm{R}}\right)$. Here $\chi_{\|}=v_{\mathrm{Te}} / k_{\|}$ is used for calculating $\chi_{\|}$and $v_{\mathrm{Te}}=\left(T_{\mathrm{e}} / m_{\mathrm{e}}\right)^{1 / 2}$ is the electron thermal velocity [21]. It should be noted that the precise form of $\chi_{\|}$for a high temperature plasma is more complex as shown in [21].

Assuming the perpendicular heat diffusivity and the plasma viscosity to be at the anomalous transport level of $0.5 \mathrm{~m}^{2} \mathrm{~s}^{-1}$, in the normalized units they are $\chi_{\perp}=\mu=$ $21\left(a^{2} / \tau_{\mathrm{R}}\right)$. The perpendicular particle diffusivity is taken to be $D_{\perp}=\chi_{\perp} / 5$. These parameters are the input for our calculations except mentioned elsewhere.

A monotonic profile of the safety factor $q$ is used, and the $q=2$ rational surface locates at $r_{\mathrm{s}}=0.628 a[8,13,15]$. The $m / n=2 / 1$ mode is stable without an externally applied helical field.

\subsection{Results from reduced MHD equations}

Before presenting the results obtained from the two fluids equations, it is useful to first have a look at the results obtained from the reduced MHD equations, which are obtained by taking $\Omega=0$ in equation (1), so that the temperature and density perturbations have no effect on the tearing mode. In this case, equations (1) and (2) are decoupled from (3)-(5).

In figure 1 the nonlinear growth of a $m / n=2 / 1$ magnetic island driven by the externally applied helical field is plotted for $\Omega_{\mathrm{E}}=1.0 \times 10^{6}$ (solid curve) and $3.0 \times 10^{6}$ (dotted), where the normalized island width $w / a$ is shown as a function of the normalized (to $\tau_{\mathrm{R}}$ ) time $t . \Omega_{\mathrm{E}}=0$ corresponds to zero equilibrium plasma rotation velocity, and $\Omega_{\mathrm{E}}=1.0 \times 10^{6}$ corresponds to a poloidal plasma rotation velocity $v_{\mathrm{p}}=-8.4 \times 10^{4}\left(a / \tau_{\mathrm{R}}\right)$ at $r_{\mathrm{s}}$. The island width is calculated from the conventional formula $w=4\left(\psi_{2 / 1} / \psi_{0}^{\prime \prime}\right)^{1 / 2}$ at the rational surface [1]. The applied helical field has a frequency $\omega_{\mathrm{f}}=0$ and an amplitude of $\psi_{\mathrm{a}}=10^{-5} a B_{0 \mathrm{t}}$. It is seen that the island grows to a larger amplitude $w=0.04 a$

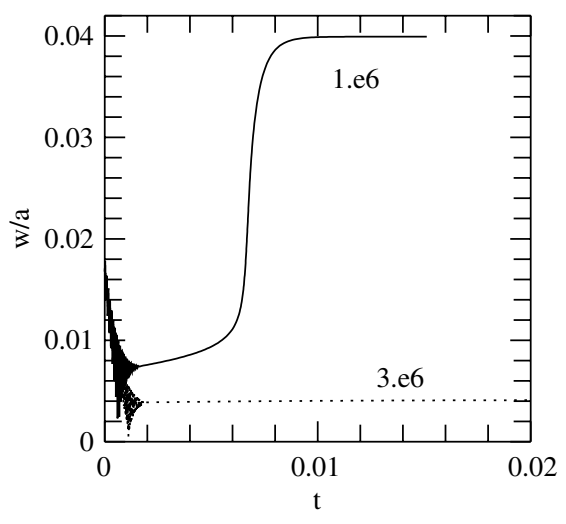

Figure 1. The normalized island width $w / a$ versus the normalized (to $\tau_{\mathrm{R}}$ ) time $t$ for $\Omega_{\mathrm{E}}=1.0 \times 10^{6}\left(-\right.$ ) and $3.0 \times 10^{6}(\cdots \cdots)$. $\Omega_{\mathrm{E}}=0$ and $1.0 \times 10^{6}$ correspond to a poloidal plasma rotation velocity $v_{\mathrm{p}}=0$ and $-8.4 \times 10^{4}\left(a / \tau_{\mathrm{R}}\right)$ at the rational surface. The applied helical field has a frequency $\omega_{\mathrm{f}}=0$ and an amplitude $\psi_{\mathrm{a}}=10^{-5} a B_{0 \mathrm{t}}$.



Figure 2. Corresponding to figure 1, the time evolution of the mode frequency (in units of $\tau_{\mathrm{R}}^{-1}$ ) is given for $\Omega_{\mathrm{E}}=1.0 \times 10^{6}$ and $3.0 \times 10^{6}$.

for a smaller rotation speed but remains at a low value for a faster rotation.

The corresponding time evolution of the normalized mode frequency is shown in figure 2 for $\Omega_{\mathrm{E}}=1.0 \times 10^{6}$ and $3.0 \times 10^{6}$. At the beginning $(t=0)$ the mode frequency is determined by the equilibrium poloidal plasma rotation, $\omega=-m v_{\mathrm{p}} / r$. In the steady state the mode is locked to the helical field for both cases, so that the mode frequency becomes zero being the same as that of the static helical field.

In figure 3 the radial profiles of the $m / n=2 / 1$ component of $\psi, \psi_{2 / 1}$, in steady state are shown for $\Omega_{\mathrm{E}}=1.0 \times 10^{6}$ and $3.0 \times 10^{6}$, where the solid (dotted) curve represents the real (imaginary) part. For a smaller rotation speed the $\psi_{2 / 1}$ profile looks like that of a usual tearing mode, while for a large rotation speed $\psi_{2 / 1}$ decreases to a very small value at the rational surface. The much smaller imaginary part compared with the real one of $\psi_{2 / 1}$ for the penetrated case indicates a small phase shift between the mode and the external field. The corresponding $m / n=2 / 1$ component of the current density perturbation, $j_{2} / 1$, is shown in figure 4 , where the solid (dotted) curve represents the real (imaginary) part. For a large rotation speed the current density perturbation has a negative peak at the 




Figure 3. Corresponding to figure 1, the radial profiles of $\psi_{2 / 1}\left(a B_{0 t}\right)$ in steady state for $\Omega_{\mathrm{E}}=1.0 \times 10^{6}$ and $3.0 \times 10^{6}$ are given. The solid (dotted) curve is the real (imaginary) part.

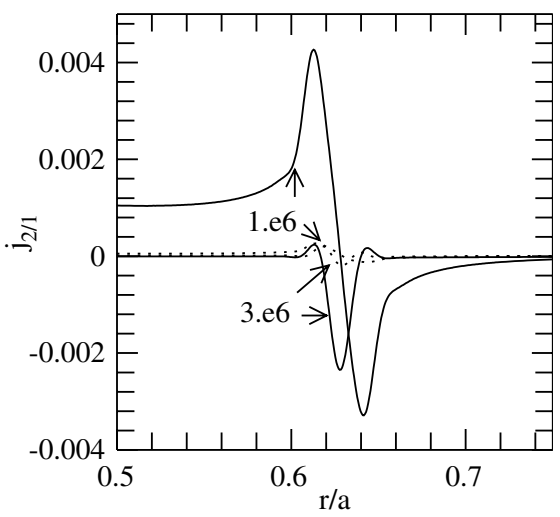

Figure 4. Corresponding to figure 3 , the $m / n=2 / 1$ component of the current density perturbation in units of $B_{0 t} / a$. The solid (dotted) curve represents the real (imaginary) part.

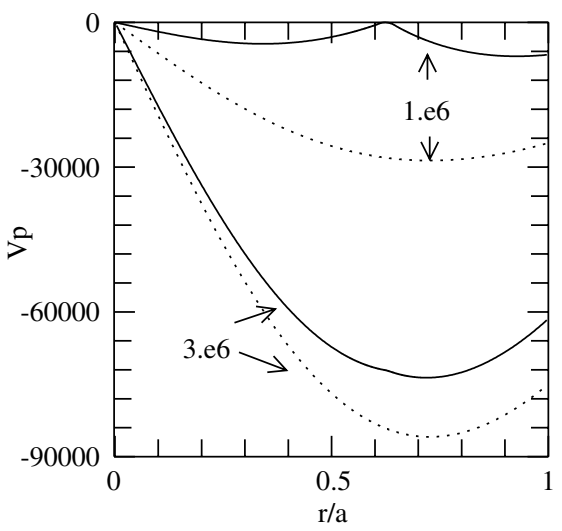

Figure 5. Corresponding to figure 3, radial profiles of the poloidal rotation velocity $v_{\mathrm{p}}\left(a / \tau_{\mathrm{R}}\right)$ for $\Omega_{\mathrm{E}}=1.0 \times 10^{6}$ and $3.0 \times 10^{6}$. The solid (dotted) curves show the steady state results (the original equilibrium ones).

rational surface, which produces an opposite field to cancel the externally applied field from the magnetic axis to the rational surface as seen in figure 3 .

In figure 5 the radial profiles of the poloidal plasma rotation velocity $v_{\mathrm{p}}$ are shown for $\Omega_{\mathrm{E}}=1.0 \times 10^{6}$ and $3.0 \times 10^{6}$. The solid curves show the results in steady state, whereas the dotted curves show the original equilibrium poloidal plasma rotation velocity profiles. For a smaller $\Omega_{\mathrm{E}}$, the field is penetrated, and $v_{\mathrm{p}}$ is brought to zero at the rational surface. For a larger rotation speed, however, $v_{\mathrm{p}}$ only slightly decreases due to the smaller magnetic island as seen in figures 1 and 3 .

In figure $6(a)$ the island width in the steady state is shown as a function of $\Omega_{\mathrm{E}}$ with $\omega_{\mathrm{f}}=0$ and $\psi_{\mathrm{a}}=10^{-5} a B_{0 \mathrm{t}}$. Only for a sufficiently small $\Omega_{\mathrm{E}}$ with $\Omega_{\mathrm{E}}<1.53 \times 10^{6}$, the helical field is penetrated, as indicated by the significantly larger saturated island width. The island width is at a very low level for a larger $\Omega_{\mathrm{E}}$, i.e. a larger plasma rotation speed.

Compared with analytical theories, the mode penetration results shown above are found to correspond to the viscoresistive regime [11,12]. Equation (42) of [12] leads to a mode penetration threshold at $\psi_{2 / 1}(r=a)=0.75 \times 10^{-5}$ with $\Omega_{\mathrm{E}}=1.53 \times 10^{6}$ and the same input data, being close to the numerical results.

Corresponding to figure $6(a)$, in $6(b)$ the ratio between the new poloidal velocity in the steady state with the error field and the original equilibrium poloidal velocity without the error field, $v_{\mathrm{p}} / v_{\mathrm{p} 0}$, at the rational surface is shown. The parameter $v_{\mathrm{p}} / v_{\mathrm{p} 0}$ sharply decreases to zero after mode penetration. Just before penetration $v_{\mathrm{p}} / v_{\mathrm{p} 0}=0.465$, being close to the analytical prediction of $v_{\mathrm{p}} / v_{\mathrm{p} 0}=0.5$ [12].

The corresponding relative phase between the magnetic perturbation at the rational surface and the error field, $\Delta \varphi$, is shown in figure $6(c) . \Delta \varphi$ decreases to nearly zero after mode penetration. Just before penetration $\Delta \varphi=89.7^{\circ}$, being approximately the same as the analytical prediction of $\Delta \varphi=90^{\circ}[11,12]$. It is seen that the analytical theories of the visco-resistive regime $[11,12]$ essentially agree with the numerical results.

\subsection{Results from two fluids equations}

In two fluids theory the equilibrium temperature and the density gradient lead to an additional mode frequency due to the diamagnetic drift (see equation (1)), such that the mode frequency is determined by $\omega=-m v_{\mathrm{p}} / r+\omega_{\mathrm{e}^{*}}$, where $\omega_{\mathrm{e}^{*}}=\Omega m\left(1 / L_{T}+1 / L_{n}\right) / r$ is the electron diamagnetic drift frequency in normalized units and $L_{T}$ and $L_{n}$ are the local gradient length of the electron temperature and density at the rational surface. In figure 7 the island width is shown as a function of $\Omega_{\mathrm{E}}$ by the solid curve for $\omega_{\mathrm{f}}=0$ and $\psi_{\mathrm{a}}=10^{-5} a B_{0 \mathrm{t}}$. The dotted curve is the same as that of figure 6 obtained for $\Omega=0$. Compared with the dotted curve, the mode penetration region shown by the solid one is shifted from the region around $\Omega_{\mathrm{E}}=0$ to a region around finite $\Omega_{\mathrm{E}}$, because the mode frequency $\omega=-m v_{\mathrm{p}} / r+\omega_{\mathrm{e}^{*}}=\omega_{\mathrm{f}}=0$ at the location shown by the vertical arrow. The dashed curve is obtained by neglecting the temperature gradient in Ohm's law. Due to the absence of the diamagnetic drift contributed from the temperature gradient, the shift of the resonance region from $\Omega_{\mathrm{E}}=0$ is smaller compared with the solid curve. It is also obvious that the island width obtained from the two fluids equations is smaller than that obtained with the reduced MHD approximation after mode penetration. This is due to the stabilizing role of the ion polarization current associated with the diamagnetic drift [22-25].

Using a rotating helical field with $\omega_{\mathrm{f}}=-2.2 \times 10^{5} / \tau_{\mathrm{R}}$ and $\psi_{\mathrm{a}}=10^{-5} a B_{0 \mathrm{t}}$, the island width is shown as a function of $\Omega_{\mathrm{E}}$ 

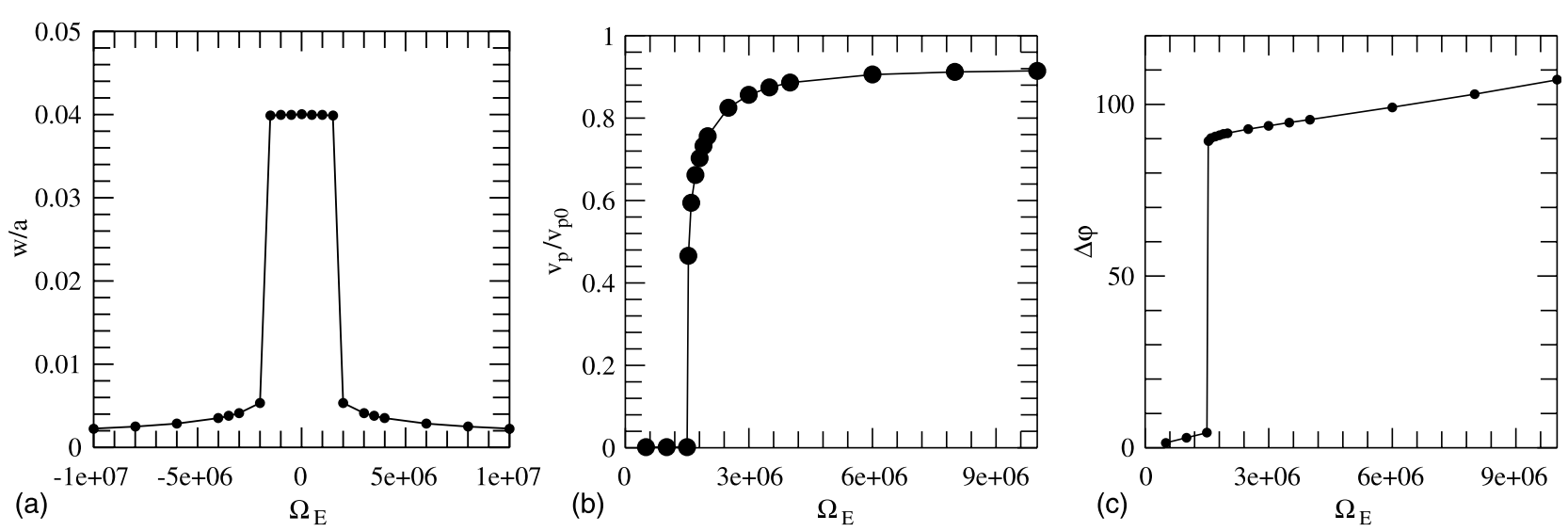

Figure 6. (a) The island width in steady state versus $\Omega_{\mathrm{E}}$, with $\omega_{\mathrm{f}}=0$ and $\psi_{\mathrm{a}}=10^{-5} a B_{0 \mathrm{t}}$. The helical field is penetrated for $\Omega_{\mathrm{E}}<1.53 \times 10^{6}$. (b) Corresponding to $(a)$, the poloidal velocity in the steady state over the original equilibrium velocity, $v_{\mathrm{p}} / v_{\mathrm{p} 0}$, at the rational surface. Just before penetration $v_{\mathrm{p}} / v_{\mathrm{p} 0}=0.465$. (c) Corresponding to $(a)$, the relative phase between the magnetic perturbation at the rational surface and the error field, $\Delta \varphi$. Just before penetration $\Delta \varphi=89.7^{\circ}$.

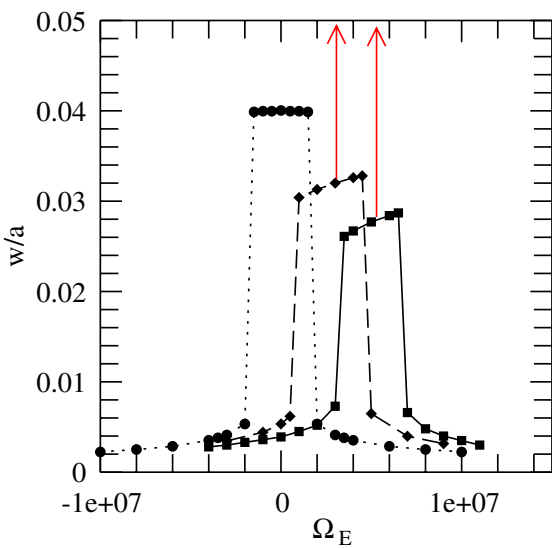

Figure 7. The island width in the steady state versus $\Omega_{\mathrm{E}}$ obtained from the two fluids equations for $\omega_{\mathrm{f}}=0$ and $\psi_{\mathrm{a}}=10^{-5} a B_{0 \mathrm{t}}(-)$. The dotted curve is the same as that of figure 6 obtained for $\Omega=\omega_{\mathrm{f}}=0$. For the dashed curve the temperature gradient in Ohm's law is neglected. The vertical arrows show the $\Omega_{\mathrm{E}}$ where the mode frequency $\omega=0$.

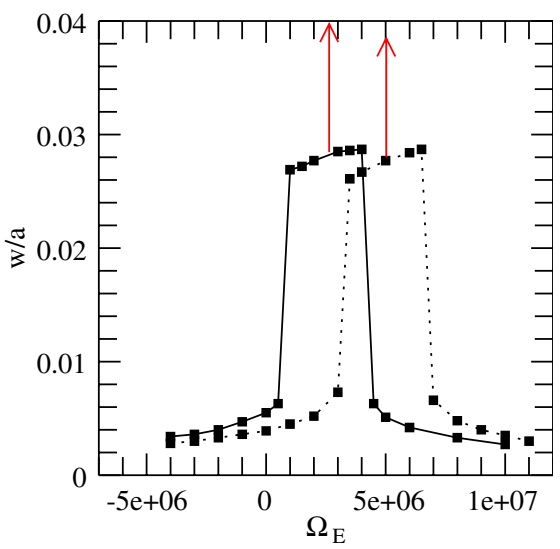

Figure 8. The island width versus $\Omega_{\mathrm{E}}$ for $\omega_{\mathrm{f}}=-2.2 \times 10^{5}$ ( $(-)$ and $\omega_{\mathrm{f}}=0(\cdots \cdots)$, with $\psi_{\mathrm{a}}=10^{-5} a B_{0 \mathrm{t}}$. The two vertical arrows show the $\Omega_{\mathrm{E}}$ value at which $\omega=\omega_{\mathrm{f}}$.

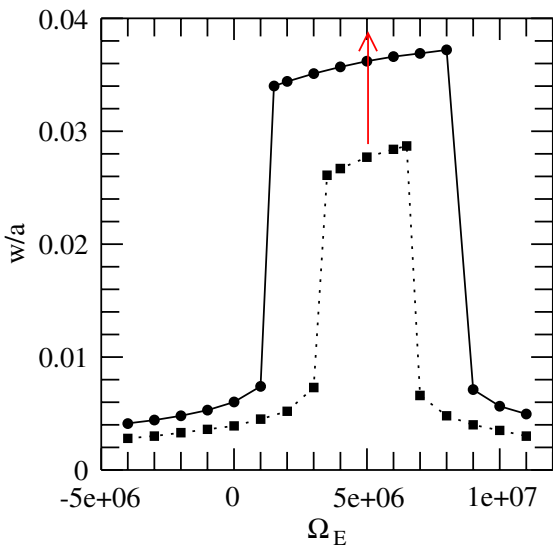

Figure 9. The island width versus $\Omega_{\mathrm{E}}$ for $\psi_{\mathrm{a}}=2 \times 10^{5}$ ( $\longrightarrow$ ) and $1 \times 10^{5}(\cdots \cdots)$, with $\omega_{\mathrm{f}}=0$. The vertical arrow shows the $\Omega_{\mathrm{E}}$ value at which $\omega=\omega_{\mathrm{f}}$.

in figure 8 by the solid curve. The dotted curve is obtained for a static helical field $\omega_{\mathrm{f}}=0$. The mode penetration is shifted to a smaller $\Omega_{\mathrm{E}}$ region for a finite frequency of the helical field as expected, since $\omega=\omega_{\mathrm{f}}$ there. The two arrows show the $\Omega_{\mathrm{E}}$ value for which $\omega=\omega_{\mathrm{f}}$ is fulfilled. A corresponding shift of the mode penetration region using a rotating helical field is observed in TEXTOR experiments [8].

Increasing the amplitude of the helical field, the mode penetration region becomes wider as seen from figure 9 , where the island width is shown as a function of $\Omega_{\mathrm{E}}$ for $\psi_{\mathrm{a}}=2 \times 10^{-5}$ (solid) and $1 \times 10^{-5} a B_{0 \mathrm{t}}$ (dotted) with $\omega_{\mathrm{f}}=0$. The vertical arrow again shows the $\Omega_{\mathrm{E}}$ value at which $\omega=\omega_{\mathrm{f}}$. A larger amplitude of the helical field is obviously required for mode penetration if the difference between mode frequency and that of the helical field becomes larger.

The island width is shown as a function of $\psi_{\mathrm{a}}$ in figure 10 for $\Omega_{\mathrm{E}}=3 \times 10^{6}$ with $\omega_{\mathrm{f}}=0$ by the solid curve. With the increase in $\psi_{\mathrm{a}}$, there is a sudden jump in the island width at $\psi_{\mathrm{a}}=1.1 \times 10^{-5} a B_{0 \mathrm{t}}$, indicating the required $\psi_{\mathrm{a}}$ for mode penetration. It should be mentioned that such a threshold does not exist if the mode frequency equals the helical field frequency. The dotted curve in figure 10 is obtained for 


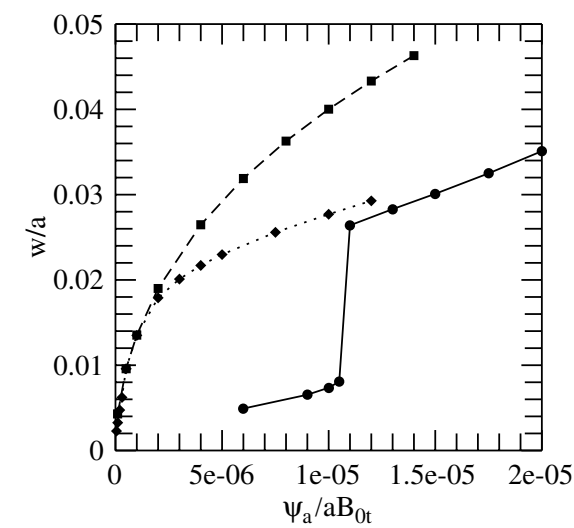

Figure 10. The island width versus $\psi_{\mathrm{a}}$ for $\Omega_{\mathrm{E}}=3 \times 10^{6}$ and $\omega_{\mathrm{f}}=0(-)$. The dotted curve is obtained for $\Omega_{\mathrm{E}}=5 \times 10^{6}$ and $\omega=-m v_{\mathrm{p}} / r+\omega_{\mathrm{e}^{*}}=\omega_{\mathrm{f}}=0$. The dashed curve is obtained from the reduced MHD equations with $\omega=\omega_{\mathrm{f}}=0$.

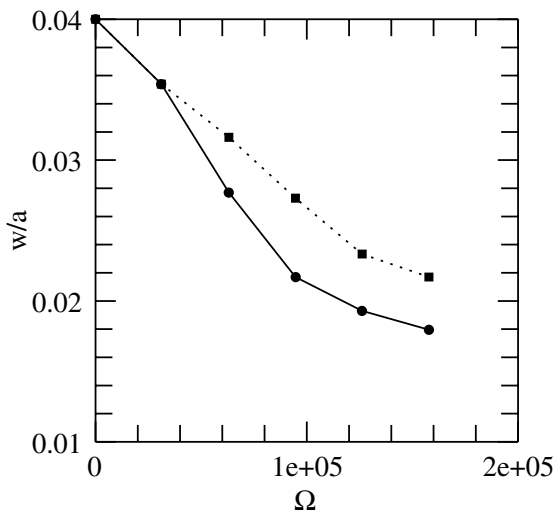

Figure 11. The island width versus $\Omega$ with $\psi_{\mathrm{a}}=10^{-5} a B_{0 \mathrm{t}}$ ( - ). The equilibrium plasma rotation is selected such that $\omega=\omega_{\mathrm{f}}=0$. The dotted curve is obtained by neglecting the electron temperature gradient in Ohm's law.

$\Omega_{\mathrm{E}}=5 \times 10^{6}$ and $\omega=-m v_{\mathrm{p}} / r+\omega_{\mathrm{e}^{*}}=\omega_{\mathrm{f}}=0$. The dashed curve is the island width obtained from the reduced MHD equations with no plasma rotation and $\omega_{\mathrm{f}}=0$. In these two cases, the island width gradually increases with increasing $\psi_{\mathrm{a}}$. Figure 10 also indicates that for the same $\psi_{\mathrm{a}}$, the island width obtained from the two fluids equations is significantly smaller than that obtained from the reduced MHD equations.

In figure 11 the island width is shown as a function of $\Omega$ with $\omega_{\mathrm{f}}=0$ and $\psi_{\mathrm{a}}=10^{-5} a B_{0 \mathrm{t}}$ (solid). The equilibrium plasma rotation is selected such that the mode frequency is zero. The dotted curve is obtained by neglecting the electron temperature gradient in Ohm's law. The island width decreases for a larger $\Omega$ (or $\omega_{e *}$ ), showing the increasing stabilizing role for a larger diamagnetic drift frequency.

The required field amplitude for mode penetration is shown as a function of $\omega_{\mathrm{p}} / \omega_{\mathrm{e}^{*}}$ in figure 12 for a constant $\Omega$, $\Omega=6.3 \times 10^{4}$, where $\omega_{\mathrm{p}}=m v_{\mathrm{p}} / r$ is the (negative) frequency due to the poloidal plasma rotation. There is a minimum required field amplitude for mode penetration at $\omega_{\mathrm{p}} / \omega_{\mathrm{e}^{*}}=1$ where $\omega=\omega_{\mathrm{f}}=0$ due to the cancelling between the equilibrium plasma rotation frequency and the diamagnetic drift frequency. The required field amplitude for

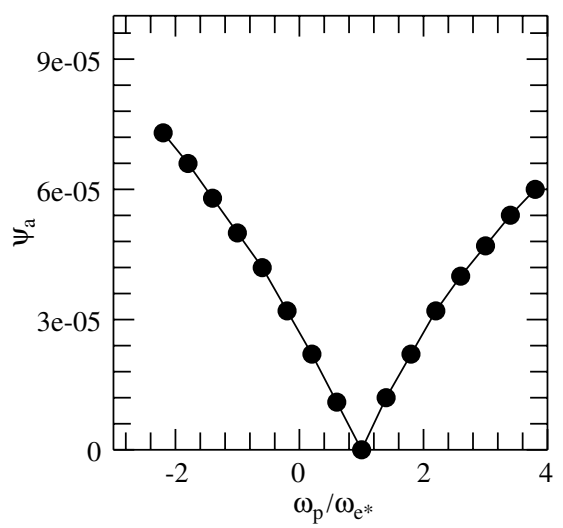

Figure 12. The required field amplitude for mode penetration versus $\omega_{\mathrm{p}} / \omega_{\mathrm{e}}$ for $\Omega=6.3 \times 10^{4}$ and $\omega_{\mathrm{f}}=0$, where $\omega_{\mathrm{p}}=m v_{\mathrm{p}} / r$ is the (negative) frequency due to poloidal plasma rotation. There is a minimum required field amplitude for mode penetration at $\omega_{\mathrm{p}} / \omega_{\mathrm{e}^{*}}=1$, where $\omega=\omega_{\mathrm{f}}=0$ due to the cancelling between the plasma rotation frequency and the diamagnetic drift frequency.

mode penetration increases as the mode frequency deviates from the frequency of the externally applied field.

It should be mentioned that the mode penetration is defined here according to the radial profile of $\psi_{2 / 1}$ in the steady state and the sudden increase in the island width with increasing $\psi_{\mathrm{a}}$ as seen from figures 3 and 10 . The penetrated island width is in fact very small for a small amplitude of $\psi_{\mathrm{a}}$ around $\omega \cong \omega_{\mathrm{f}}$.

In TEXTOR experiments the equilibrium plasma rotation is in the toroidal direction rather than in the poloidal one $[8,9]$. To calculate the mode penetration in the plasma with a toroidal rotation, an additional equation would be required to calculate the nonlinear evolution of the toroidal rotation velocity. Since in our model, equations (1)-(5), only the poloidal rotation is included, a much larger $\left(\times 10^{2}\right)$ plasma viscosity, $\mu=2.1 \times 10^{3}\left(a^{2} / \tau_{\mathrm{R}}\right)$, is used in the following numerical calculations to simulate the toroidal rotation effect. Such an approximated approach is based on the following two reasons $[1,2]$ : (a) the electromagnetic force to slow down the plasma rotation in the toroidal direction is smaller by a factor of $(n / m)\left(r_{\mathrm{s}} / R\right)$ than that in the poloidal direction. (b) To have the same mode frequency due to plasma rotation, the toroidal rotation requires a speed which is $(m / n)\left(R / r_{\mathrm{s}}\right)$ times larger than the poloidal one [1,2]. These two effects lead to a relative larger viscous force compared with the electromagnetic force for the toroidal rotation case by a factor of $\left[(m / n)\left(R / r_{\mathrm{s}}\right)\right]^{2}$, which is of the order of $10^{2}$. Therefore, with only the equilibrium poloidal rotation in our model, a larger plasma viscosity gives a more reasonable balance between the electromagnetic and the viscous forces.

The numerical results obtained with $\mu=2.1 \times 10^{3}\left(a^{2} / \tau_{\mathrm{R}}\right)$ are shown in figure 13, in which the required field amplitude for mode penetration is shown as a function of $\omega_{\mathrm{p}} / \omega_{\mathrm{e}^{*}}$, with the other parameters being the same as those for figure 12 . Similarly to figure 12 , there is a minimum in the required field amplitude for mode penetration at $\omega_{\mathrm{p}} / \omega_{\mathrm{e}^{*}}=1$. The required field amplitude, however, shows asymmetry on the two sides of the minimum, being larger for $\omega_{\mathrm{p}} / \omega_{\mathrm{e}^{*}}>1$ than that for $\omega_{\mathrm{p}} / \omega_{\mathrm{e}^{*}}<1$. For a large plasma viscosity, the nonlinear change in the plasma rotation due to the electromagnetic force is less significant, while the diamagnetic drift frequency 


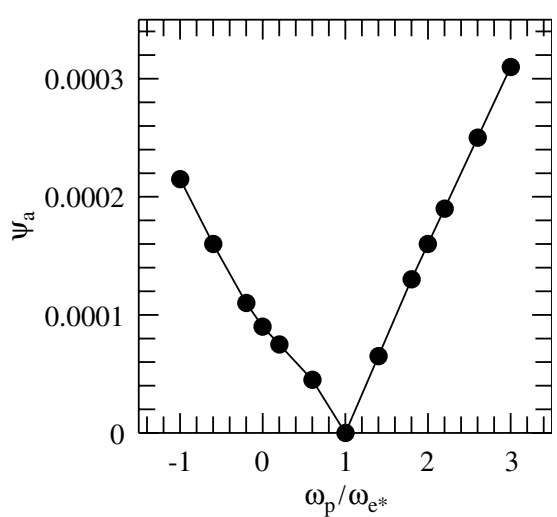

Figure 13. The required field amplitude for mode penetration versus $\omega_{\mathrm{p}} / \omega_{\mathrm{e}^{*}}$, with $\mu=2.1 \times 10^{3}\left(a^{2} / \tau_{\mathrm{R}}\right)$ and other parameters being the same as those for figure 12. The required amplitude is asymmetric on the two sides of $\omega_{\mathrm{p}} / \omega_{\mathrm{e}^{*}}=1$, being larger for $\omega_{\mathrm{p}} / \omega_{\mathrm{e}^{*}}>1$.

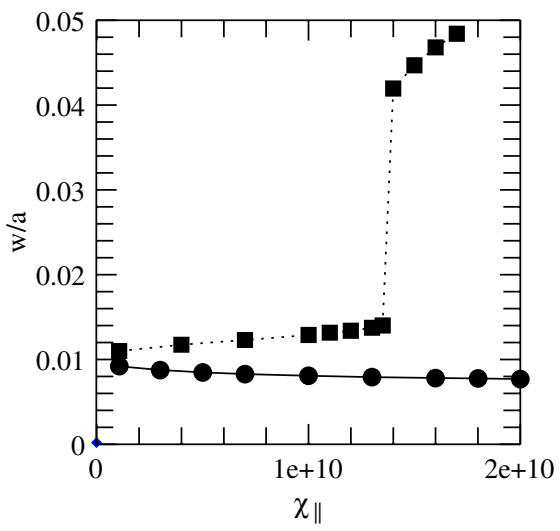

Figure 14. The nonlinear saturated island width versus $\chi_{\|}\left(a^{2} / \tau_{\mathrm{R}}\right)$ for $\left(\omega_{\mathrm{p}} / \omega_{\mathrm{e}^{*}}-1\right)=2 / 5(-)$ and $-2 / 5(\cdots \cdots)$, with $\psi_{\mathrm{a}}=3 \times 10^{-5} a B_{0 \mathrm{t}}$ and the other parameters being the same as those for figure 13. With increasing $\chi_{\|}$the island width decreases for $\omega_{\mathrm{p}} / \omega_{\mathrm{e}^{*}}>1$ but increases for $\omega_{\mathrm{p}} / \omega_{\mathrm{e}^{*}}<1$. The field is penetrated when $\chi_{\|}>1.4 \times 10^{10} a^{2} / \tau_{\mathrm{R}}$ for $\left(\omega_{\mathrm{p}} / \omega_{\mathrm{e}^{*}}-1\right)=-2 / 5$.

decreases due to the parallel heat transport with increasing applied field amplitude. Therefore, the difference between the mode frequency and the applied field frequency increases for $\omega_{\mathrm{p}} / \omega_{\mathrm{e}^{*}}>1$ but decreases for $\omega_{\mathrm{p}} / \omega_{\mathrm{e}^{*}}<1$. This causes the asymmetry in the required field amplitude for mode penetration as observed in TEXTOR experiments $[8,9]$. While for a small plasma viscosity, the mode penetration occurs before the field amplitude is large enough to significantly change the diamagnetic drift frequency, leading to the required field amplitude being approximately symmetrical on the two sides of $\omega_{\mathrm{p}} / \omega_{\mathrm{e}^{*}}=1$ as shown in figure 12. Comparing figure 12 with figure 13 it is also clear that the mode penetration threshold is significantly higher with a larger plasma viscosity (or toroidal rotation) as expected $[1,2]$.

The above explanation for the asymmetry in the mode penetration threshold can be seen from the effect of the parallel heat diffusivity $\chi_{\|}$. In figure 14 the nonlinear saturated island width is shown as a function of $\chi_{\|}$(in units of $a^{2} / \tau_{\mathrm{R}}$ ) for $\left(\omega_{\mathrm{p}} / \omega_{\mathrm{e}^{*}}-1\right)=2 / 5$ (solid curve) and $-2 / 5$ (dotted), with $\psi_{\mathrm{a}}=$ $3 \times 10^{-5} a B_{0 \mathrm{t}}$ and the other parameters being the same as those for figure 13. For lower $\chi_{\|}, \chi_{\|} \sim 10^{9}$, the island widths are about the same for $\left(\omega_{\mathrm{p}} / \omega_{\mathrm{e}^{*}}-1\right)=2 / 5$ and $-2 / 5$, indicating the symmetry on the two sides of $\left(\omega_{\mathrm{p}} / \omega_{\mathrm{e}^{*}}-1\right)=0$ as seen from figure 12. With increasing $\chi_{\|}$the island width decreases for $\omega_{\mathrm{p}} / \omega_{\mathrm{e}^{*}}>1$ but increases for $\omega_{\mathrm{p}} / \omega_{\mathrm{e}^{*}}<1$. The field is penetrated at $\chi_{\|}=1.4 \times 10^{10} a^{2} / \tau_{\mathrm{R}}$ for $\left(\omega_{\mathrm{p}} / \omega_{\mathrm{e}^{*}}-1\right)=-2 / 5$ as indicated by a much larger island width. The increase in $\chi_{\|}$ leads to a stronger flattening of the local electron temperature profile around the rational surface due to the faster parallel heat transport and a corresponding larger change in the diamagnetic drift frequency, so that the frequency difference between the mode and the applied field becomes larger for $\omega_{\mathrm{p}} / \omega_{\mathrm{e}^{*}}>1$ but smaller for the opposite case. The results of figures 13 and 14 indicate that the asymmetry in the required field amplitude for mode penetration depends on the parallel heat transport, which is in turn affected by the helical field amplitude and the balance between the electromagnetic and the viscous force.

Comparing with analytical theory it is seen that for the case with low plasma viscosity (figures 7 and 12), the mode penetration threshold is similar to that from the MHD results $[11,12]$. In this case only a frequency shift in the penetration region is observed due to the contribution of the diamagnetic frequency to the mode frequency, and the penetration threshold is approximately symmetric on the two sides of the minimum, as predicted by analytical theory that the mode penetration threshold obtained from the two fluids equations is similar to that from the reduced MHD equations, if the mode frequency due to background plasma rotation is replaced by that due to the diamagnetic drift [14]. The heat diffusive layer width at the rational surface, $W_{\mathrm{c}}=a\left(\chi_{\perp} / \chi_{\|}\right)^{1 / 4}\left[8 L_{q} /(\varepsilon a n)\right]^{1 / 2}[26,27]$, is found to be $W_{\mathrm{c}}=0.039 a$ in this case, where $L_{q}=q / q^{\prime}$ and $\varepsilon=a / R$. While the island width $w$ before mode penetration is smaller than $0.01 a$, leading to $w<1 / 4 W_{\mathrm{c}}$. Therefore, the assumption that the diamagnetic frequency is not changed before mode penetration is essentially valid [14].

The difference between the present results and that of [14] is seen from figures 13 and 14. With a larger plasma viscosity to model the toroidal rotation, the island width before mode penetration is about $w=0.02-0.03 a$ (depending on the plasma rotation frequency), being closer to $W_{\mathrm{c}}$. In this case the parallel heat transport affects the mode penetration threshold as seen from figure 14 . For $\left(\omega_{\mathrm{p}} / \omega_{\mathrm{e}^{*}}-1\right)=-2 / 5$ the mode penetration occurs at $\chi_{\|}=1.35 \times 10^{10}\left(a^{2} / \tau_{\mathrm{R}}\right)$, corresponding to $W_{\mathrm{c}}=0.021 a$, being only 1.5 times larger than the island width $w=0.014 a$ just before mode penetration. This indicates that when the unpenetrated island width is close to $W_{\mathrm{c}}, w / W_{\mathrm{c}}$ is also an important parameter in determining the penetration threshold, leading to the asymmetry in the required field amplitude on the two sides of $\omega_{\mathrm{p}} / \omega_{\mathrm{e}^{*}}=1$ as shown in figure 13. In [15] the temperature change and the nonlinear effect are not considered, and in [14] the parallel heat conduction is neglected.

\section{Discussion and summary}

Numerical modelling of the error field penetration has been carried out based on the two fluids equations in a periodical cylindrical geometry. The electron temperature and density perturbations are self-consistently calculated. This extends the previous results from reduced MHD equations and the fourfield model [10-15]. 
Comparing the results obtained from the two fluids equations with those from the reduced MHD equations, there are three major differences: the first difference is in the mode frequency. The mode differs from the fluid velocity by the electron diamagnetic drift in two fluids theory. The second difference is in the nonlinear penetrated island width, which decreases with increasing diamagnetic drift frequency. The third difference is the asymmetry of the mode penetration threshold on the two sides of $\omega_{\mathrm{p}} / \omega_{\mathrm{e}^{*}}=1$, when using a large plasma viscosity to model the toroidal rotation effect. The required field amplitude for mode penetration is large for $\omega_{\mathrm{p}} / \omega_{\mathrm{e}^{*}}>1$. The first and the third features of the two fluids results have been observed on TEXTOR $[8,9]$, and the first feature is also seen from the previous results $[14,15]$.

For a fusion reactor the plasma rotation velocity is expected to be low, since neutral beam injection (NBI) heating is not needed for a burning plasma. In this case, the diamagnetic drift frequency is expected to be important in determining the mode penetration threshold as it may be comparable to or even larger than the plasma rotation frequency. Since the electron temperature of a fusion reactor is higher than that of the existing tokamaks, the nonlinear change in the diamagnetic drift frequency would be more significant due to the faster parallel heat transport.

It should be noticed that the toroidal rotation effect is simulated here only by a large plasma viscosity. A consistent approach including the nonlinear evolution of the toroidal plasma rotation would be very helpful for comparison with the present results. The cold ion assumption used in this present paper is not generally satisfied for tokamak plasmas. When the bootstrap current perturbation is further included, leading to the NTM, the mode penetration threshold and the penetrated island width would be different. The NTM usually grows to a large amplitude once it is excited [17]. Further studies are required to include these effects. Nevertheless, the basic features observed in TEXTOR experiments, such as the shift of the mode penetration region from balanced NBI to unbalanced $\mathrm{NBI}$ due to the electron diamagnetic drift, the larger required field amplitude for mode penetration for $\omega_{\mathrm{p}} / \omega_{\mathrm{e}^{*}}>1$ than that for $\omega_{\mathrm{p}} / \omega_{\mathrm{e}^{*}}<1$ and the significant increase in the mode penetration threshold when $\omega$ is different from $\omega_{\mathrm{f}}[8]$, are found from the present results.

In summary, numerical modelling of the error field penetration has been carried out based on the two fluids equations. It is found that there is a minimum required field amplitude for mode penetration when the mode frequency is the same as the externally applied helical field frequency. The mode penetration threshold increases as the mode frequency deviates from the field frequency and is asymmetric on the two sides of the minimum value. For the plasma rotation velocity being faster than the electron diamagnetic drift, the required field amplitude is larger. Such an asymmetry is evident for the plasma with a large plasma viscosity (to model the toroidal ration effect) and becomes more significant with increasing parallel heat conductivity. The penetrated island width decreases for a larger electron diamagnetic drift frequency.

\section{References}

[1] Nave M.F.F. and Wesson J.A. 1990 Nucl. Fusion 302575

[2] Hender T.C. et al 1992 Nucl. Fusion 322091

[3] Buttery R.J. et al 2000 Nucl. Fusion 40807

[4] Buttery R.J. et al 1999 Nucl. Fusion 391827

[5] La Haye R.J. et al 1992 Phys. Fluids B 42098

[6] La Haye R.J., Hyatt A.W. and Scoville J.T. 1992 Nucl. Fusion 322119

[7] Wofle S.W. et al 2005 Phys. Plasmas 12056110

[8] Koslowski H.R. et al 2006 Nucl. Fusion 46 L1

[9] Finken K.H. et al 2005 Phys. Rev. Lett. 94015003

[10] Fitzpatrick R. and Hender T.C. 1991 Phys. Fluids B 3644

[11] Fitzpatrick R. 1993 Nucl. Fusion 331049

[12] Fitzpatrick R. 1998 Phys. Plasmas 53325

[13] Kikuchi Y. et al 2006 Plasma Phys. Control. Fusion 48169

[14] Cole A. and Fitzpatrick R. 2006 Phys. Plasmas 13032503

[15] Kikuchi Y. et al 2006 Phys. Rev. Lett. 97085003

[16] Yu Q., Günter S. and Scott B.D. 2003 Phys. Plasmas 10797

[17] Yu Q., Günter S. and Lackner K. 2004 Phys. Plasmas 11140

[18] Yu Q., Günter S., Giruzzi G., Lackner K. and Zabiego M. 2000 Phys. Plasmas 7312

[19] Günter S., Yu Q., Krüger J. and Lackner K. 2005 J. Comput. Phys. 209354

[20] Yu Q. 2006 Phys. Plasmas 13062310

[21] Chang Z. and Callen J.D. 1992 Phys. Fluids B 41167

[22] Smolyakov A.I. 1993 Plasma Phys. Control. Fusion 35657

[23] Wilson H.R., Connor J.W., Hastie R.J. and Hegna C.C. 1996 Phys. Plasmas 3248

[24] Connor J.W., Waelbroeck F.L. and Wilson H.R. 2001 Phys. Plasmas 82835

[25] Sauter O. et al 1997 Phys. Plasmas 41654

[26] Fitzpatrick R. 1995 Phys. Plasmas 2825

[27] Hölzl M., Günter S., Yu Q. and Lackner K. 2007 Phys. Plasmas 14052501 